\title{
Endovascular Repair of Traumatic Subclavian Artery Injuries: A Single- Center Experience
}

Barão T, Queiroz AB*, Apoloni R, Ricardo A and Nelson DL

University of São Paulo Medical School, Division of Vascular and Endovascular Surgery, São Paulo, Brazil

\begin{abstract}
Subclavian artery injury is a life-threatening condition and represents a challenge for trauma surgeons. Open surgical treatment often requires wide surgical exposures; including thoracotomy thus endovascular repair has emerged as a minimally invasive alternative. We report our single-center recent experience of fifteen patients with subclavian artery injuries, all of them treated by endovascular techniques. There were 9 blunt (60\%) and 6 penetrating (40\%) traumas and we had immediate operative success in all cases. Three patients presented with complete artery transections and were treated with a through-and-through technique. We believe endovascular treatment of subclavian artery injuries is a safe technique and can be performed in different types of trauma.
\end{abstract}

\section{Keyword: Endovascular; Subclavian artery; Trauma}

\section{Introduction}

Subclavian artery injury is a challenging complication of blunt and penetrating trauma. The open surgical repair is often difficult requiring wide surgical access and dissection in traumatized areas. The subclavian artery is protected by the subclavius muscle, the clavicle, the first rib, the deep cervical fascia and the costo-coracoid ligament [1] Open surgery represents the classical management, but it is associated with high morbidity mostly because the need of extensive exposures, which often requires clavicular resection, thoracotomy or median sternotomy.

When active bleeding, pseudoaneurysm or critical limb ischemia is present, urgent repair is required. Arterial rupture usually causes lifethreatening hemorrhages, and must be carefully ruled out by physical examination as well as diagnostic imaging [2].

In recent years, increasing number of reports has described the successful management of vascular injuries by way of endovascular route. Covered stents have emerged as a valuable alternative to open surgery in subclavian injuries with encouraging results [3-9]. This report describes a series of patients with traumatic subclavian artery injury treated with an endovascular approach in a single center.

\section{Patients and Methods}

We report a retrospective review of our experience with endovascular repair of subclavian injuries (not including iatrogenic lesions) in which all patients underwent endovascular approach. The acquired data were prospectively entered into an electronic database, but medical records were also reviewed retrospectively in order to retrieve missing baseline data.

All patients were admitted at the trauma unit of our hospital, directly or being transferred from other centers. A multidisciplinary approach was used in the care of these patients; mainly involving the vascular surgery, anesthesia and radiology departments.

All procedures were performed in the operating room, under general anesthesia and orotracheal intubation. The entire setting was prepared for an endovascular procedure and for an open repair if necessary. Fluoroscopic monitoring was used in all patients with a mobile C-arm (OEC 9900; GE, USA or BV Pulsera; Philips, USA).

In this series, no intravenous heparin bolus was administered before stent implantation and bleeding control, confirmed by angiographic imaging. Even in patients without obvious hemorrhage, heparin was not administered because of the risk of bleeding by venous injury and other associated traumas.

Initially there was not a standardization technique and the procedure was not exactly the same in all patients. Although, with the growing experience acquired over the cases we managed to standardize the procedure.

All patients received prophylactic pre-procedural antibiotic treatment. The patient was positioned supine and the upper limb in the side injured was positioned at a 90 to the body.

The first step in all cases was the obtaining of a percutaneous right femoral access with a short introducer sheath. Using a hydrophilic 0.035 guidewire (Aqualiner; Nipro Corp., SP, Brazil) and with the aid of a 5F catheter (Vert or Head Hunter; Cook Inc., IN, USA) the target vessel was reached - left or right subclavian artery. At this moment an angiography was performed to determine the extent of the lesion. The catheter was replaced by a 7 to $9 \mathrm{~mm}$ diameter over-the-wire (OTW) balloon-catheter (Mustang, Boston Corp., MA, USA) and the balloon was inflated if continuous bleeding was present in angiography or hemodynamic instability arose.

The hydrophilic wire was used to gentle transposes the lesion and if it was possible, the wire was exchanged by a stiffer one (Amplatz; Cordis Corp. FL, USA). A centimeter-sizing catheter (Pig Tail; Cook Inc., IN, USA) was used to determine the length of the lesion and the artery diameter if there was no previous computed tomography (CT) scan. The covered stent was chosen to reach 10 to $15 \%$ oversizing and to cover at least 1,5 to $2 \mathrm{~cm}$ of healthy artery proximal and distal to the

*Corresponding author: Queiroz AB, University of São Paulo Medical School, Division of Vascular and Endovascular Surgery, São Paulo, Brazil, Tel: +55 11 3091-3116; E-mail: Andrebrito01@hotmail.com

Received July 30, 2015; Accepted November 10, 2015; Published November 18,2015

Citation: Barão T, Queiroz AB, Apoloni R, Ricardo A, Nelson DL (2015) Endovascular Repair of Traumatic Subclavian Artery Injuries: A Single-Center Experience. J Vasc Med Surg 3: 229. doi:10.4172/2329-6925.1000229

Copyright: $\odot 2015$ Barão T, et al. This is an open-access article distributed unde the terms of the Creative Commons Attribution License, which permits unrestricted use, distribution, and reproduction in any medium, provided the original author and source are credited. 
injury. The stent accommodation was performed with a same diameter OTW catheter-balloon using gentle pressure (Figures 1A and 1B).

If there was complete arterial transection and the guidewire progression reached the hematoma or the pleural cavity, a brachial cut down access was obtained in the injured side. A snare (ENSnare - Angiotech, FL, USA or Indy OTW Vascular Retriever - Cook, Bjaeverskov, Denmark) was introduced through this access and captured the previous introduced hydrophilic guidewire allowing a trough-and-trough brachial-femoral access. This maneuver can often be time consuming and challenging requiring patience and dexterity. The covered stent was then chosen and released as previously described. Completion angiography was performed to assure pervious graft and bleeding control (Figures 2A-2C).

\section{Results}

Fifteen patients ( 13 men; age range $16-51$; mean age 32 years; 2 women, age range 19-70; mean age 44 years) admitted in the emergency of Clinics Hospital of São Paulo, in a period of 3 years, with subclavian artery trauma were treated during the first 8 hours after admission, by endovascular covered stent placement.

We had nine blunt $(60 \%)$ and six penetrating $(40 \%)$ traumas of the subclavian artery. The cause of the injury was traffic accident in 7 patients $(46,6 \%)$ - motorcycle in $5(33,3 \%)$, car in $1(6,6 \%)$, and bike in

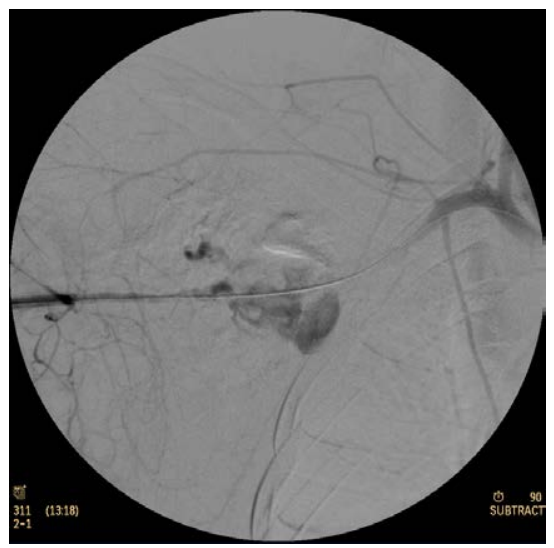

Figure 1A: The covered stent of healthy artery proximal.

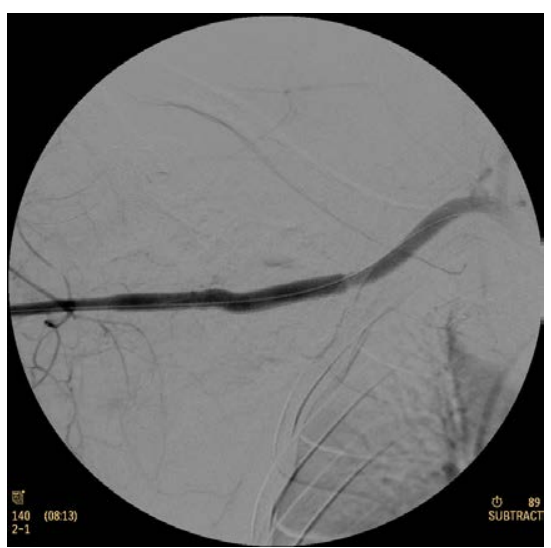

Figure 1B: The covered stent was accommodate with catheter-balloon using gentle pressure.

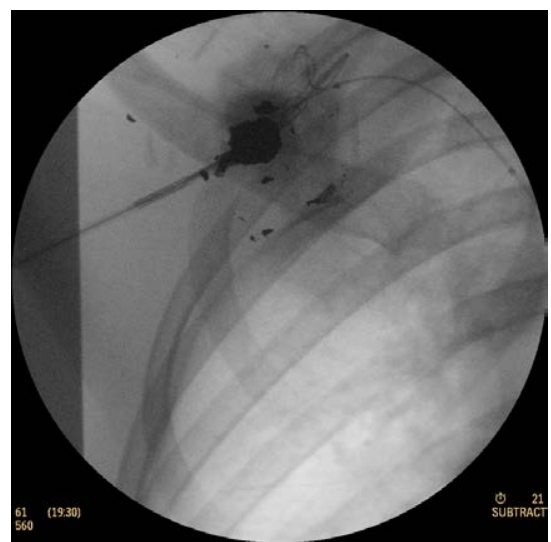

Figure 2A: Complete arterial transection and the guidewire progression of pleural cavity.

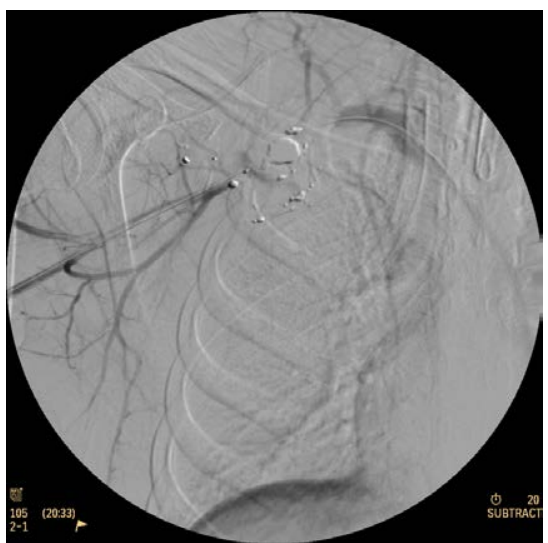

Figure 2B: The capturing of the hydrophilic guide wire allowing a trough-andtrough brachial-femoral access.

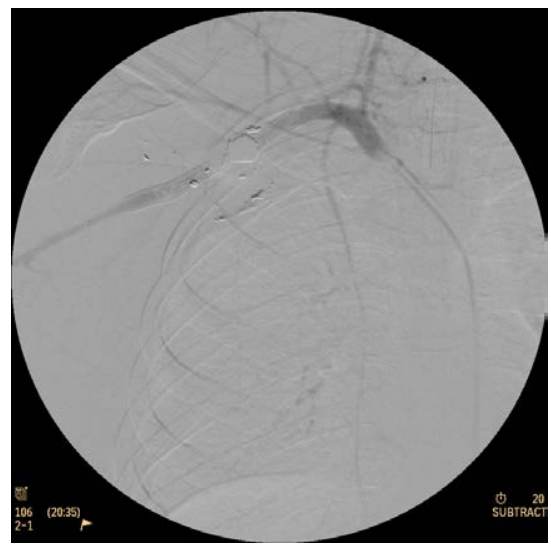

Figure 2C: Angiography completion to assure pervious graft and bleeding control.

$1(6,6 \%)$; gunshot injury in $5(33,3 \%)$; stab wound in $1(6,6 \%)$; fall from 4 meters in $1(6,6 \%)$; and a work-related accident in $1(6,6 \%)$.

Twelve patients had a contrast-enhanced CT scanning in the emergency before the procedure, and the others had just clinical 
diagnosis and went to the operating room for a catheter based angiography and simultaneous endovascular treatment.

Surgical access was only femoral in most cases (12/15). At the totally transected artery injuries (3/15) we used a combined access, femoral and brachial. The self-expandable covered stents were Wallgraft (Boston Scientific, MA, USA) in the seven initial cases $(46,6 \%)$ and Viabahn (Gore, AZ, USA) in the last eight cases $(53,3 \%)$.

In traumas with complete transection of the artery, we had the combined access to overpass the lesion using the snare through the brachial artery to capture the guidewire coming from the femoral.

The three totally transected arteries were due to penetrating trauma in two and to blunt trauma in one patient. In one of the initial cases we were not using an occlusive catheter-balloon in the proximal artery and we had massive bleeding to the thorax with cardiac arrest when the trough-and-trough access was obtained, but we had successfully deployed the covered stent and the patient recovered without repeating the same procedure.

All cases were successfully treated with the lesion covered by the stent graft and adequate reperfusion of the arm. We had one acute brachial artery thrombosis during the procedure that was solved with a Fogarty catheter trombectomy in the same setting.

Placement of the stent-graft was technically successful in all patients. In all cases, the pseudoaneurysm was completely excluded, the bleeding stopped and the injured arterial segment reconstructed. In three patients the vertebral artery origin was occluded by the graft due to the proximal location of the injury, but there was no cerebral periprocedural event in this series. None of the patients required conversion to open surgery. Eight patients had hemothorax or pnemothorax in the ipsilateral side of the artery injury and required thorax drainage. Plexus injury was present in six patients.

There were two deaths in the perioperative period at four and twenty seven days after the procedure due to other lesions. There were no vascular access site-related complications and no endograft infections during hospital stay and during follow-up. One patient had occlusion of the stent at six monthly follow-up but was asymptomatic and revascularization was not required.

\section{Discussion}

Subclavian arterial injuries account for only 2 to $5 \%$ of acute vascular injuries and most vascular surgeons have limited expertise in their endovascular management $[10,11]$. The overwhelming majority of these injuries are from penetrating trauma. According to the literature a minority - 1 to $5 \%$, of all subclavian artery injuries results from blunt trauma and are usually associated with fractures or dislocations $[11,12]$. A history of trauma with bone injury should raise the index of suspicion. Physical examination of the upper limb must focus on skin color, temperature sensation, hand motility as well as radial pulse. Shoulder hematoma is also often present.

Contrast enhanced CT has an important role in diagnosing and surgical planning because it can reveal where the lesion is and its extension. Moreover, it is usually readily available in trauma care centers. In our institution this exam is available just beside the emergency room and can be performed quickly after admission.

Surgical exposure for the treatment of traumatic subclavian injury can be challenging and treacherous and carries a high morbidity [13]. It can be done either by supraclavicular approach with or without clavicular resection, by thoracotomy, median sternotomy or by combined access for larger exposures. Regardless of surgical access, there is a potential risk of subclavian vein and brachial plexus injuries. The reported mortality in some series ranges between $10 \%$ and $30 \%$ [14]. In addition, it carries a high risk of major bleeding [15].

In an effort to avoid the morbidity and mortality associated with open approach, the focus has shifted to endovascular techniques. Parodi successfully reported the endovascular repair of a subclavian injury using an endovascular approach in 1995 when a covered stent was deployed to treat a traumatic arteriovenous fistula [16]. Widespread use of endovascular treatment has become an acceptable and less invasive alternative to surgical repair in these injuries.

The main advantage of endovascular approach is that the system can be inserted through a remote access site, obviating the need for extensive surgical exposure. Recognized advantages include diminished pain, decreased disability and rapid recovery.

Improved covered stents flexibility, reduced fracture potential, and a greater variety of lengths and diameters have increased the versatility for use in axillary and subclavian locations (Figure 3). We believe that newer self-expanding devices, composed of nitinol stents covered with polytetrafluoroethylene, are more flexible and conform more easily to the vessel walls. Therefore, we thought that these devices appear to be suitable especially in these cases. Embolic complications during placement due to dissection and embolization of the devices may occur but were not present in our experience.

We believe endovascular repair of subclavian injuries is safe and can be done in different types of lesions. At any time of the surgery the control of the bleeding can be achieved with an occlusive ballooncatheter in the proximal subclavian artery. Furthermore comparing with the open approach, we can potentially minimize the damage of surrounding structures and reduce the blood loss.

With the advances in endovascular devices technology and with the growing experience acquired, more anatomically difficult lesions can be repaired using an endoluminal approach, including complete arterial transection. In our experience we decided to begin the procedures by a percutaneous femoral access because we thought it is less invasive than the cut down brachial access, even with the use of larger sheaths to the deployment of the covered stents. Furthermore this enables quick access placement of a hemostatic catheter-balloon proximal to the lesion, which could not be achieved through the arm. The brachial artery was reserved only for patients with complete artery transection and requiring a trough-and-trough access. We had immediate success

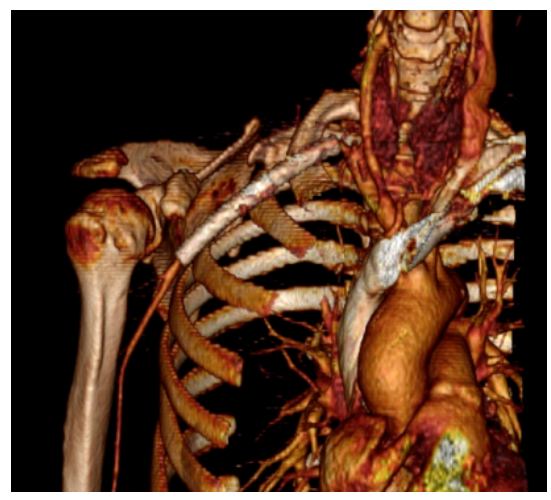

Figure 3: Axillary and subclavian locations. 
rate of $100 \%$ in blunt and penetrating injuries, with only two related perioperative events: a reversed cardiac arrest and a brachial artery thrombosis solved at the same procedure.

Subacute thrombosis and intimal hyperplasia leading to in-stent stenosis or vessel occlusion are other possible complications associated with covered stents and the long-term patency of these devices is still unknown. However, a later revascularization can be done if necessary in a less emergent situation and after the acute problem has been solved.

The subclavian artery trauma is a serious and potentially fatal injury and the endovascular approach has been established as a feasible and benefical technique. Vascular surgeons in trauma centers must be familiar with this approach and covered stents should be available on the shelf in these hospitals.

\section{References}

1. Kendall KM, Burton JH, Cushing B (2000) Fatal subclavian artery transection from isolated clavicle fracture. J Trauma 48: 316-318.

2. Stokkeland PJ, Soreide K, Fjetland L (2007) Acute endovascular repair of right subclavian arterial perforation from clavicular fracture after blunt trauma. $J$ Vasc Interv Radiol 18: 689-690.

3. Patel AV, Marin ML, Veith FJ, Kerr A, Sanchez LA (1996) Endovascular graft repair of penetrating subclavian artery injuries. J Endovasc Surg 3: 382-388.

4. Xenos ES, Freeman M, Stevens S, Cassada D, Pacanowski J, et al. (2003) Covered stents for injuries of subclavian and axillary arteries. J Vasc Surg 38: 451-454

5. Krishnan KG, Martin KD, Schackert G (2008) Traumatic lesions of the brachial plexus: an analysis of outcomes in primary brachial plexus reconstruction and secondary functional arm reanimation. Neurosurgery 62: 873-885.
6. Becker GJ, Benenati JF, Zemel G, Sallee DS, Suarez CA, et al. (1991) Percutaneous placement of a balloon-expandable intraluminal graft for lifethreatening subclavian arterial hemorrhage. J Vasc Interv Radiol 2: 225-229.

7. McKinley AG, Carrim AT, Robbs JV (2000) Management of proximal axillary and subclavian artery injuries. Br J Surg 87: 79-85.

8. du Toit DF, Strauss DC, Blaszczyk M, de Villiers R, Warren BL (2000) Endovascular treatment of penetrating thoracic outlet arterial injuries. Eur $\mathrm{J}$ Vasc Endovasc Surg 19: 489-495.

9. Bukhari HA, Saadia R, Hardy BW (2007) Urgent endovascular stenting of subclavian artery pseudoaneurysm caused by seatbelt injury. Can J Surg 50: 303-304.

10. Rich NM, Hobson RW, Jarstfer BS, Geer TM (1973) Subclavian artery trauma. J Trauma 13: 485-496.

11. Graham JM, Feliciano DV, Mattox KL, Beall AC Jr, DeBakey ME (1980) Management of subclavian vascular injuries. J Trauma 20: 537-544

12. Lim LT, Saletta JD, Flanigan DP (1979) Subclavian and innominate artery trauma. A five-year experience with 17 patients. Surgery 86: 890-897.

13. Demetriades D, Asensio JA (2001) Subclavian and axillary vascular injuries. Surg Clin North Am 81: 1357-1373, xiii.

14. Posner MP, Deitrick J, McGrath P, Mendez-Picon G, Sobel M, et al. (1988) Nonpenetrating vascular injury to the subclavian artery. J Vasc Surg 8: 611 617.

15. Renger RJ, de Bruijn AJ, Aarts HC, van der Hem LG (2003) Endovascular treatment of a pseudoaneurysm of the subclavian artery. J Trauma 55: 969971.

16. Parodi JC (1995) Endovascular repair of abdominal aortic aneurysms and other arterial lesions. J Vasc Surg 21: 549-555. 